\title{
Demographic features, BMPR2 status and outcomes in distal chronic thromboembolic pulmonary hypertension
}

\author{
Jay Suntharalingam, Rajiv D Machado, Linda D Sharples, Mark R Toshner, Karen K Sheares, Rodney \\ J Hughes, David P Jenkins, Richard C Trembath, Nicholas W Morrell, Joanna Pepke-Zaba
}

See end of article for authors' affiliations

\section{Correspondence to: Dr Joanna Pepke-Zaba, Pulmonary Vascular Diseases Unit, Papworth Hospital NHS Trust, Papworth Everard, Cambridgeshire CB3 8RE, UK; Joanna.pepkezaba@ papworth.nhs.uk}

Received 22 August 2006 Accepted 1 January 2007 Published Online First 7 February 2007

\begin{abstract}
Background: Although pulmonary endarterectomy (PEA) is potentially curative in chronic thromboembolic pulmonary hypertension (CTEPH), some patients have distally distributed disease that is not amenable to surgery. The aetiology and characteristics of this patient group are currently not well understood.

Objectives: This study compares the baseline demographic features and outcomes in subjects with distal CTEPH, those with proximal CTEPH and those with idiopathic pulmonary arterial hypertension (IPAH) to determine whether these conditions represent separate entities or whether they exist along the same spectrum of disease.

Methods: The medical history, clinical characteristics, bone morphogenetic protein receptor type II (BMPR2) mutation status and outcomes of 96 subjects with IPAH, 35 with distal CTEPH and 68 with proximal CTEPH referred to a single specialist centre between 1994 and 2005 were reviewed.

Results: There were significant differences between the distal CTEPH, proximal CTEPH and IPAH groups in age $(55.9$ years vs 54.8 years vs 46.2 years, $\mathrm{p}<0.001)$, proportion who were male $(43 \%$ vs $69 \%$ vs $29 \%$, $p<0.001)$, previous deep vein thrombosis $(28.6 \%$ vs $30.9 \%$ vs $3.1 \%, p<0.001)$, positive BMPR 2 status $(0 \%$ vs $0 \%$ vs $15 \%, p=0.018)$, mean pulmonary artery pressure $(47.3 \mathrm{~mm} \mathrm{Hg}$ vs $45.4 \mathrm{~mm} \mathrm{Hg}$ vs $54.8 \mathrm{~mm} \mathrm{Hg}$, $\mathrm{p}<0.001$ ) and total pulmonary resistance (12.9 WU vs $12.4 \mathrm{WU}$ vs $18.1 \mathrm{WU}, \mathrm{p}<0.001)$. Patients with distal CTEPH and those with IPAH were managed similarly and had comparable survival characteristics ( 1 year survival $77 \%$ vs $86 \% ; 3$ year survival $53 \%$ vs $60 \% ; p=0.68$ ).

Conclusions: Patients with distal CTEPH share certain demographic features with patients with proximal CTEPH that not only indicate a common aetiology but also help to differentiate them from patients with IPAH. Despite more favourable haemodynamic parameters in those with distal CTEPH, patients in this group had a poor long-term outcome which was similar to that of patients with IPAH.
\end{abstract}

hronic thromboembolic pulmonary hypertension (CTEPH) is an important cause of pulmonary hypertension that is commonly considered to be the consequence of acute pulmonary embolic disease. ${ }^{1}$ Following an acute event, unresolved residual thrombus becomes organised and fibrosed, leading to ongoing obstruction to pulmonary blood flow. Untreated, this leads to progressive pulmonary hypertension, right ventricular dysfunction and death. ${ }^{2}$ Recent evidence suggests that CTEPH may be more common than originally thought and may complicate up to $3.8 \%$ of acute pulmonary embolic events. ${ }^{3}$ In patients with predominantly "proximal" disease, surgical intervention (pulmonary endarterectomy, PEA) can be very effective, often leading to normalisation of pulmonary arterial pressures. ${ }^{4}$ However, when the degree of haemodynamic compromise is out of proportion to the quantity of surgically accessible disease, surgery is often inappropriate. ${ }^{5}$ PEA in these patients is less successful and is associated with a higher mortality. ${ }^{6}$ In this inoperable or "distal" disease, where the majority of organised thrombus lies beyond the subsegmental level, management has historically consisted of supportive treatment alone.

Idiopathic pulmonary arterial hypertension (IPAH) is characterised by a small vessel vasculopathy that occurs in the absence of a known precipitant. A distinction has recently been made between patients with this disorder and those who have both $\mathrm{PAH}$ and a positive family history (familial PAH or FPAH). ${ }^{7}$ This follows the discovery that mutations in the bone morphogenetic protein receptor type II (BMPR2) gene underlie up to $70 \%$ of cases of FPAH. ${ }^{8}$ BMPR 2 mutations have also been observed in IPAH, albeit at a lower incidence, ${ }^{9}$ a proportion of which represent de novo spontaneous mutations or hitherto undiagnosed familial disease. ${ }^{9}{ }^{10}$ Histologically, IPAH is characterised not only by intimal fibrosis and plexiform lesions but also by organised in situ thrombus. ${ }^{11}$ In situ thrombus formation in these patients can be extensive, sometimes clinically and radiologically mimicking the appearances of CTEPH. ${ }^{12}$ Moreover, in patients with CTEPH, peripheral IPAHlike vasculopathic changes have been demonstrated that coexist with the more upstream obstructive thrombotic lesions already described. ${ }^{13}$ These shared histological features have therefore led to the suggestion that IPAH and CTEPH may represent a continuum of disease, thus casting doubt over the presumed embolic aetiology of CTEPH. ${ }^{14}{ }^{15}$ In such a model, distal CTEPH could represent an overlap condition, displaying characteristics of both IPAH and proximal CTEPH.

There is a paucity of information available on the characteristics of patients with distal CTEPH, particularly compared with patients with proximal CTEPH and IPAH. Most studies have combined data on patients with distal and proximal CTEPH on

Abbreviations: BMPR2, bone morphogenetic protein receptor type II; CTEPH, chronic thromboembolic pulmonary hypertension; CTPA, CT pulmonary angiography; $\mathrm{Dm}$, pulmonary membrane diffusion capacity; DVT, deep vein thrombosis; FEV 1 , forced expiratory volume in $1 \mathrm{~s}$; FVC, forced vital capacity; FPAH, familial pulmonary arterial hypertension; $\mathrm{HRCT}$, high resolution computed tomography; IBD, inflammatory bowel disease; IPAH, idiopathic pulmonary arterial hypertension; $\mathrm{KCO}$, carbon monoxide transfer coefficient; $\mathrm{PAP}$, pulmonary artery pressure; $\mathrm{PE}$, pulmonary embolism; PEA, pulmonary endarterectomy; TLC, total lung capacity; TLCO, carbon monoxide transfer factor; VA, ventriculo-atrial; Vc, pulmonary capillary blood volume 
the assumption that the two conditions represent the same disease entity. ${ }^{216}$ This study compares the baseline demographic features and outcomes of subjects with IPAH, distal CTEPH and proximal CTEPH to help characterise the aetiology of each of these conditions further. This will also help determine whether these conditions represent separate entities or whether they, in fact, exist along the same spectrum of disease.

\section{METHODS \\ Subjects}

The study was undertaken at the Pulmonary Vascular Diseases Unit (PVDU), Papworth Hospital, one of five specialist pulmonary hypertension centres in the UK, and the national referral centre for PEA surgery. All consecutive patients referred with IPAH or CTEPH between 1994 and 2005 were identified. All had been diagnosed with pulmonary hypertension at right heart catheterisation using standard diagnostic criteria. ${ }^{7}$ All subjects underwent a standardised imaging protocol that has been published elsewhere. ${ }^{17}$ This relies primarily on multislice CT pulmonary angiography (CTPA) and high resolution CT (HRCT) scanning to distinguish between patients with CTEPH and IPAH using a 32-slice CT scanner. The use of CTPA in diagnosing thromboembolic obstruction has previously been validated against catheter-directed pulmonary angiography. ${ }^{18}$ HRCT scanning has also been shown to be as sensitive as (and more specific than) radionuclide scanning in differentiating CTEPH from IPAH. ${ }^{19}$

Once identified, subjects with CTEPH were further characterised through a combination of catheter-directed pulmonary angiography and/or contrast-enhanced magnetic resonance pulmonary angiography to assess for operability. All imaging was reviewed by a panel of specialist physicians, radiologists and surgeons who determined the distribution of disease. Only subjects with distal disease (ie, disease where thrombotic lesions were situated predominantly or exclusively beyond the subsegmental level) were included for analysis in the distal group, thus excluding patients who were inoperable for reasons other than disease distribution alone. Subjects were only included in the proximal group if they had undergone PEA surgery and demonstrated normalisation of their pulmonary artery pressures (PAP; mean PAP $<25 \mathrm{~mm} \mathrm{Hg}$ ) at their 3month postoperative assessment; this approach was taken to exclude subjects with coexisting proximal and distal disease. DNA was obtained from subjects in all three groups, where available, and analysed for the presence of BMPR2 mutations by direct fluorescent sequencing on an ABI3730 using previously described primers and conditions. ${ }^{8}$

Approval to conduct the study was granted by the local review board. Ethical approval was granted and patient consent obtained before testing for BMPR2 mutations.

\section{Data collection}

Baseline demographic data were obtained by retrospective review of case notes. Subjects were only deemed to have had a prior thromboembolic event if a deep vein thrombosis (DVT) or pulmonary embolus (PE) had been demonstrated on appropriate imaging (Doppler leg ultrasound or venography for DVT and ventilation-perfusion scanning or CTPA for PE) $>6$ months before referral and managed appropriately with formal anticoagulation. Data were also collected on the presence of established risk factors for CTEPH including splenectomy, the presence of a ventriculo-atrial (VA) shunt, osteomyelitis and inflammatory bowel disease (IBD). ${ }^{20}$ Subjects were considered to have received targeted medical treatment if they had been prescribed a prostanoid, an endothelin receptor antagonist or a phosphodiesterase inhibitor during the follow-up period.

\section{Statistical analysis}

All eligible patients from this centre were studied. Continuous variables are summarised as mean (SD) values. Normally distributed variables were compared between groups using a one-way ANOVA followed by post-hoc pairwise comparisons for significant differences using Bonferroni corrections. Categorical variables were summarised as the percentage of the total studied and compared using the $\chi^{2}$ test or Fisher's exact test whenever low expected cell counts were encountered. Data were complete with the exception of BMPR2 status. Survival rates from presentation at the specialist centre were estimated using Kaplan-Meier methods, treating death as an event and censoring all other subjects at the end of follow-up or at transplantation, whichever occurred first. A comparison of survival between the distal CTEPH and IPAH groups was made using a log-rank test.

All analyses were performed using SPSS V12 software; p values $<0.05$ were considered significant.

\section{RESULTS}

\section{Baseline characteristics}

The inclusion criteria for the study were fulfilled by 199 subjects, 96 of whom had IPAH, 35 had distal CTEPH and 68 had proximal CTEPH. BMPR2 mutation status was available in 90 subjects (45\%). A comparison of the baseline characteristics is shown in table 1 . The IPAH group was significantly younger and significantly less likely to have had a previous thromboembolic event than the distal and proximal CTEPH groups. The proportion of men was significantly higher in the proximal CTEPH group than in either the IPAH or distal CTEPH groups. The prevalence of splenectomy also differed significantly between the three populations, with the highest prevalence occurring in the distal CTEPH group. There was no difference in the incidence of VA shunt, osteomyelitis or IBD between the groups. The distribution of BMPR2 mutations was significantly different between the three groups; mutations were present in $15 \%$ of subjects with IPAH but were not observed in either the distal CTEPH or proximal CTEPH groups.

Table 2 summarises the haemodynamic and pulmonary function for the three groups. Mean PAP and total pulmonary resistance (TPR) were significantly higher in the IPAH group than in the distal and proximal CTEPH groups. Carbon monoxide transfer factor (TLCO) was significantly higher in the proximal CTEPH group than in the IPAH or distal CTEPH groups. Carbon monoxide transfer coefficient (KCO) was significantly higher in the proximal CTEPH group than in the IPAH group, and forced vital capacity (FVC) was lower in the distal CTEPH group than in the proximal CTEPH group. There were no clinically relevant differences between the groups in terms of either haematological or biochemical results.

\section{Outcomes}

Follow-up data were complete for all subjects, with none lost to follow-up. $93.8 \%$ of the patients with proximal CTEPH improved symptomatically to WHO functional class I or II by the time of their 3-month postoperative assessment following PEA surgery. During subsequent follow-up two subjects died, both of presumed cardiorespiratory causes. Actuarial survival in this highly selected group of subjects with proximal CTEPH was $98.5 \%, 97 \%$ and $97 \%$ respectively at 1,3 and 5 years.

A similar proportion of subjects in both the distal CTEPH and IPAH groups received targeted treatment (68\% vs 66\%) during follow-up. First-line treatments used included intravenous epoprostenol, intravenous iloprost, subcutaneous trepostinil, nebulised iloprost, bosentan and sildenafil. At 12 months, actuarial survival was $86 \%$ in subjects with IPAH compared with $77 \%$ in those with distal CTEPH. The IPAH group 
Table 1 Baseline characteristics of the IPAH, distal CTEPH and proximal CTEPH groups

\begin{tabular}{|c|c|c|c|c|}
\hline & $\begin{array}{l}\text { IPAH } \\
(n=96)\end{array}$ & $\begin{array}{l}\text { Distal CTEPH } \\
(\mathbf{n}=35)\end{array}$ & $\begin{array}{l}\text { Proximal CTEPH } \\
(\mathrm{n}=68)\end{array}$ & p Value \\
\hline Mean (SD) age (years)* & $46.2(16.9)$ & $55.9(13.1)$ & $54.8(14.6)$ & $<0.001$ \\
\hline Male sext & $29 \%$ & $43 \%$ & $69 \%$ & $<0.001$ \\
\hline Mean (SD) BMI $\left(\mathrm{kg} / \mathrm{m}^{2}\right)$ & $27.1(5.8)$ & $27.9(7.5)$ & $27.4(3.9)$ & 0.77 \\
\hline Positive BMPR2 status $\ddagger$ & $15 \%(n=40)$ & $0 \%(n=25)$ & $0 \%(n=25)$ & 0.018 \\
\hline Prior TED $\$$ & $5.2 \%$ & $74.3 \%$ & $75 \%$ & $<0.001$ \\
\hline Prior DVT & $3.1 \%$ & $28.6 \%$ & $30.9 \%$ & $<0.001$ \\
\hline Previous splenectomy** & $3.1 \%$ & $11.4 \%$ & $1.6 \%$ & 0.042 \\
\hline Positive smoking history & $47.9 \%$ & $65.7 \%$ & $50 \%$ & 0.18 \\
\hline \multicolumn{5}{|l|}{ WHO functional class } \\
\hline ॥ & $20(21 \%)$ & $10(29 \%)$ & $11(16 \%)$ & \\
\hline III & 50 (53\%) & $17(49 \%)$ & $32(47 \%)$ & \\
\hline IV & $24(26 \%)$ & $8(22 \%)$ & $25(37 \%)$ & 0.37 \\
\hline Mean (SD) 6MWD (m) & $257(118)$ & $254(99)$ & $256(131)$ & 1.00 \\
\hline \multicolumn{5}{|c|}{ 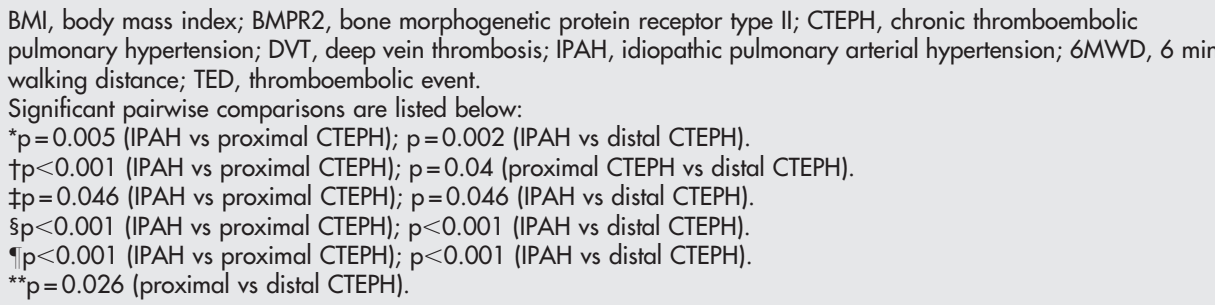 } \\
\hline
\end{tabular}

continued to have better survival rates 3 years after presentation ( $60 \%$ vs $53 \%$ ), but survival rates were similar to the distal CTEPH group at 5 years (48\% vs 53\%). Overall, there was no statistically significant difference in survival between these two groups $(\mathrm{p}=0.68$, fig 1$)$.

\section{DISCUSSION}

This study shows that patients with distal CTEPH and those with proximal CTEPH are comparable in terms of age at presentation, BMPR2 status and prior thromboembolic history, but that they differ from patients with IPAH in all these respects. Although all three groups were equally limited in functional terms, patients with IPAH had significantly worse haemodynamic parameters at presentation. Despite these differences, long-term survival was similar in both the distal CTEPH and IPAH groups.

There is both histological and clinical evidence to support a role for thrombosis in IPAH. ${ }^{1121}$ Given that other histological features are also common to both CTEPH and IPAH, ${ }^{13}$ it has been suggested that these two conditions may exist on a continuum of disease. This casts doubt over the presumed embolic origins of CTEPH and would suggest that in situ thrombosis plays a more significant role in the disease. However, if distal CTEPH represents an overlap condition, one would expect patients with distal CTEPH to share some demographic features with patients with IPAH. However, this study shows that both distal and proximal CTEPH differ from IPAH with respect to key factors such as age and prior

Table 2 Baseline haemodynamics and spirometric parameters of the IPAH, distal CTEPH and proximal CTEPH groups

\begin{tabular}{llllc}
\hline & $\begin{array}{l}\text { IPAH } \\
(\mathbf{n}=96)\end{array}$ & $\begin{array}{l}\text { Distal CTEPH } \\
(\mathbf{n}=35)\end{array}$ & $\begin{array}{l}\text { Proximal CTEPH } \\
(\mathbf{n}=68)\end{array}$ & $\begin{array}{l}\text { p Value } \\
\text { (ANOVA) }\end{array}$ \\
\hline $\begin{array}{l}\text { Haemodynamics } \\
\text { RAP }\end{array}$ & $9.5(6)$ & $10.2(7.9)$ & $8.6(5.8)$ & 0.45 \\
Cardiac index & $1.91(0.68)$ & $2.11(0.68)$ & $2.07(0.67)$ & 0.23 \\
mPAP† & $54.8(13.1)$ & $47.3(11.9)$ & $45.4(12.0)$ & $<0.001$ \\
TPR & $18.1(8.3)$ & $12.9(5.2)$ & $12.4(5.2)$ & $<0.001$ \\
Pulmonary function & & & & 0.07 \\
FEV $(\%$ predicted) & $84.0(16)$ & $74.7(22.1)$ & $80.4(18.5)$ & 0.009 \\
FVC (\% predicted)§ & $92.0(16.8)$ & $83.3(24.7)$ & $95.7(16.9)$ & 0.09 \\
TLC (\% predicted) & $98.2(15.2)$ & $90.8(19.8)$ & $95.6(15.5)$ & 0.002 \\
TLCO (\% predicted) & $66.3(19.3)$ & $62.8(13.4)$ & $75.5(17.7)$ & 0.002 \\
KCO (\% predicted)** & $78.8(22.1)$ & $85.3(17.4)$ & $91.6(19.3)$ & \\
\hline
\end{tabular}

Values are mean (SD)

CTEPH, chronic thromboembolic pulmonary hypertension; FEV 1 , forced expiratory volume in $1 \mathrm{~s}$; FVC, forced vital capacity; IPAH, idiopathic pulmonary arterial hypertension; KCO, carbon monoxide transfer coefficient; mPAP, mean pulmonary artery pressure; RAP, resting arterial pressure; TLC, total lung capacity; TLCO, carbon monoxide transfer factor; TPR, total pulmonary resistance.

Significant pairwise comparisons are listed below:

${ }^{*} \mathrm{p}=0.02$ (IPAH vs proximal CTEPH).

$t p<0.001$ (IPAH vs proximal CTEPH); $p=0.008$ (IPAH vs distal CTEPH).

$\neq p<0.001$ (IPAH vs proximal CTEPH); $p=0.001$ (IPAH vs distal CTEPH).

$\S p=0.007$ (distal CTEPH vs proximal CTEPH).

I $p=0.01$ (IPAH vs proximal CTEPH); $p=0.006$ (proximal CTEPH vs distal CTEPH).

${ }^{* *} p=0.002$ (IPAH vs proximal CTEPH). 


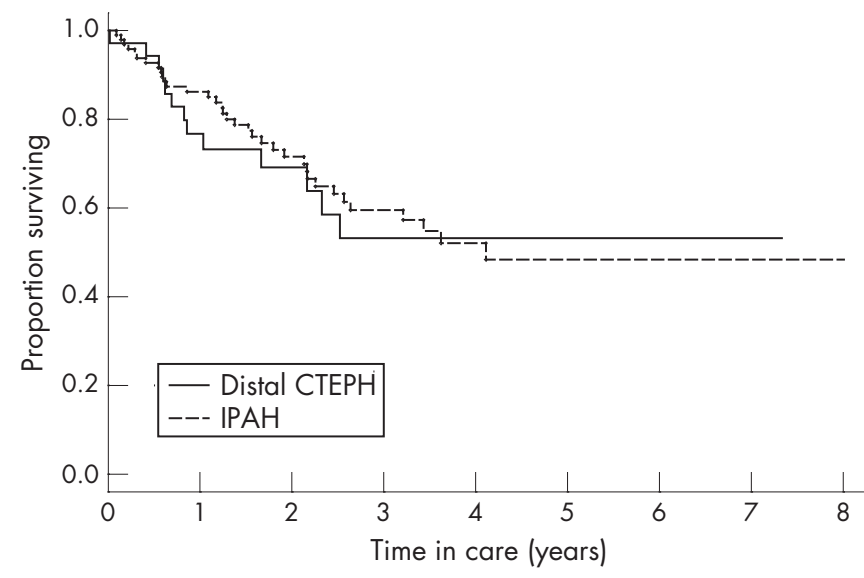

Figure 1 Kaplan-Meier survival estimates for idiopathic pulmonary arterial hypertension (IPAH) and distal chronic thromboembolic pulmonary hypertension (CTEPH) patient groups.

thromboembolic history. Moreover, there was a trend towards a higher proportion of men in both CTEPH groups compared with IPAH. The age and sex differences between patients with proximal CTEPH and those with IPAH seen here are consistent with previously reported studies, implying that our patient population is comparable to those of other centres. ${ }^{22}$ The incidence of prior DVT in patients with proximal CTEPH is likewise similar to previously published work. ${ }^{23}$ The marked difference in the incidence of DVT between the three groups found in this study therefore favours the role of a shared thromboembolic aetiology which is common to both distal and proximal CTEPH but not IPAH.

The BMPR2 receptor is a member of the transforming growth factor- $\beta$ cell signalling superfamily and plays a critical role in vascular growth, development and maintenance. ${ }^{24}$ Mutations in the BMPR2 gene underlie most cases of $\mathrm{FPAH}^{8}$ but only a proportion of cases of IPAH, ${ }^{9}$ suggesting that other genetic or environmental factors are also important in sporadic forms of the disease. BMPR2 mutations have been described in other forms of pulmonary hypertension, ${ }^{25-27}$ but the prevalence of these mutations in CTEPH has not been specifically studied to date. Although the results of this study are limited by the proportion of DNA samples available, they suggest that BMPR2 mutations are not a feature of either distal or proximal CTEPH. This provides further evidence, albeit circumstantial, that the aetiology of both of these forms of CTEPH is distinct from that of IPAH.

Despite the similarities linking distal and proximal CTEPH in this study, there was a significant difference in the prevalence of prior splenectomy between the two groups. Furthermore, splenectomy was also more common in distal CTEPH than in IPAH, albeit not significantly so. Although not new findings, these results confirm previous studies that suggest that splenectomy is more likely to be associated with distal, rather than proximal, disease. ${ }^{20}{ }^{22}$ Splenectomy appears independently to promote a prothrombotic state, possibly through loss of the filtering function of the spleen and consequent persistence of abnormal red cells. ${ }^{15}$ It is possible that splenectomy is also associated with other abnormalities of haemostasis that may particularly predispose to distal CTEPH. For example, thromboembolic material may be more fragile in these subjects, thus fragmenting more easily within the pulmonary vasculature and triggering more distally distributed disease.

Haemodynamic evaluation is essential for diagnosing pulmonary hypertension but cannot typically distinguish between the different forms of pulmonary hypertension. In this study, however, there were significant differences between the haemodynamic parameters of the two CTEPH groups and the IPAH group, despite all three groups sharing similar functional characteristics of WHO class and baseline 6 min walking distance. Although this discrepancy between the groups could be attributed to age, virtually all of the patients with proximal CTEPH returned to WHO functional class I or II following surgery, suggesting that age-related co-morbidities alone did not contribute significantly to exercise limitation. This implies that patients with CTEPH are more functionally limited than their haemodynamic measures suggest. Although PVR is commonly taken as a surrogate of disease severity, another key determinant of right ventricular afterload is pulmonary arterial impedance. This measure of the opposition to pulsatile (versus mean) components of flow is itself dependent on the mechanical properties of the proximal pulmonary arteries. ${ }^{28}$ CTEPH affects more proximal components of the pulmonary vasculature than IPAH and is thus likely to have a more adverse effect on pulmonary impedance. This would lead to more right ventricular strain-and hence more exercise limitation-than suggested by standard right heart catheterisation measurements alone.

Both restrictive and obstructive spirometric changes have previously been described in IPAH and CTEPH. ${ }^{29-31}$ Restrictive changes in CTEPH have been attributed to parenchymal scarring resulting from previous pulmonary infarction. This may explain the smaller lung volumes seen in the distal CTEPH group, who had more peripherally distributed disease, compared with the proximal CTEPH group. However, the differences in mean $\mathrm{FEV}_{1}, \mathrm{FVC}$ and TLC were small and did not alone allow clear differentiation of the three patient groups. Mean TLCO, however, was also significantly higher in the proximal CTEPH group than in the other two groups. Following correction for alveolar volume, this difference remained significant between the proximal CTEPH and IPAH groups. TLCO is a function of both pulmonary capillary blood volume $(\mathrm{Vc})$ and pulmonary membrane diffusion capacity (Dm). There are relatively few published data on Vc and Dm in pulmonary hypertension. However, as a large proportion of disease in proximal CTEPH is situated within major vessels, one would expect Dm to be relatively preserved. Moreover, an extensive bronchial collateral circulation often develops in proximal CTEPH which may help maintain Vc through retrograde perfusion. Conversely, patients with IPAH have been shown to have impairment of both $\mathrm{Vc}_{\mathrm{c}}$ and Dm, possibly as a complication of their small vessel vasculopathy. ${ }^{32}$ As there is proportionally more downstream involvement in distal CTEPH, a reduction in Vc and Dm similar in magnitude to that of IPAH would therefore be expected. Although previous work has failed to show a difference in Dm or Vc between patients with CTEPH and those with IPAH, this may have been related to the fact that no differentiation was made between distal and proximal disease. $^{32}$

The natural history of a disease is best ascertained by prospectively following a large cohort of untreated patients. However, given the rarity of distal CTEPH, this poses some logistical difficulties and would require a multicentre approach. Furthermore, there is increasing evidence, albeit from uncontrolled studies, that targeted treatment may be beneficial in these patients, possibly by modifying the IPAH-like vasculopathic changes. ${ }^{33-38}$ As such, given that there are no licensed treatments for this progressive condition, deteriorating patients are typically managed similarly to those with IPAH and treated with targeted treatments on a compassionate basis. For these reasons, prospective data on treatment-naive patients are unlikely to be forthcoming and historical outcome data must be relied on in the meantime. When examining outcome in 
CTEPH, it is important that a clear distinction is made between proximal and distal disease as management differs so markedly between the two groups. Unfortunately, the two papers that have previously studied outcomes have not made this distinction, and thus there is a paucity of information available regarding distal disease. ${ }^{216}$ Conversely, the outcome in patients with IPAH is much better described ${ }^{39}$ and we have previously been able to validate survival of our own IPAH population against known measures. ${ }^{40}$ In this study, using our IPAH population as a benchmark, we were therefore able to assess the relative survival characteristics of distal CTEPH compared with IPAH. The results show that, when both patient groups are managed in a similar manner, the outcomes are comparable. This confirms that a diagnosis of distal CTEPH conveys a poor prognosis, leading to almost $50 \%$ mortality by 3 years. There is therefore a need for more clinical trials to define more clearly the role of medical treatments in distal CTEPH. Moreover, the more favourable haemodynamic parameters in the distal CTEPH group suggest that standard haemodynamic measures alone may underestimate the prognosis in this condition. This may be relevant when considering the timing of referral for transplantation.

An obvious limitation of the study is the small number of subjects present in the distal CTEPH cohort compared with the other two groups, reflecting the relative rarity of the condition. This may have resulted in low power to detect minor or moderate differences between the groups. Another limitation is the wide variation in targeted treatments prescribed to individuals with distal CTEPH. The latter reflects the expansion in the number of treatments available for use in this specialty during the period of time studied. This is controlled, to an extent, by the fact that both patients with distal CTEPH and those with IPAH are managed alike at our institution and thus receive the most appropriate targeted treatment available at the time. A further potential drawback to the study is the diagnosis of distal CTEPH itself. There are no clear universal criteria as to what constitutes inoperable disease, and thus the threshold for offering surgery to patients with CTEPH may vary from institution to institution. The study was performed in an established PEA centre where almost 300 PEA procedures have been performed. All subjects were assessed with a minimum of two imaging modalities and discussed within a multidisciplinary setting in the presence of experienced specialist surgeons, radiologists and physicians. It is therefore hoped that the distal CTEPH cohort described in this study are representative of patients seen elsewhere. Conversely, the study benefits from being performed within a single centre, thus ensuring that a uniform diagnostic approach was taken in all patients. In addition, the group with proximal CTEPH was clearly defined by postoperative haemodynamics and therefore excluded patients who may have had coexisting proximal and distal disease at presentation.

In summary, this study shows that patients with distal CTEPH and those with proximal CTEPH share certain demographic features that not only indicate a common aetiology but also help differentiate them from patients with IPAH. Despite more favourable haemodynamic measures, patients with distal CTEPH experience levels of functional limitation similar to those with IPAH and share an equally poor long-term outcome. These results emphasise the importance of securing an early diagnosis in distal CTEPH so that appropriate management can be instituted.

\section{ACKNOWLEDGEMENTS}

The authors thank Mrs Denise Hodgkins for maintaining the database and all the healthcare providers who helped manage the patients included in the study.

\section{Authors' affiliations}

Jay Suntharalingam, Linda D Sharples, Mark R Toshner, Karen K Sheares, Rodney J Hughes, David P Jenkins, Joanna Pepke-Zaba, Papworth Hospital NHS Trust, Papworth Everard, Cambridgeshire, UK

Rajiv D Machado, Richard C Trembath, Division of Genetics and Molecular Medicine (Guy's Campus), King's College, London, UK

Linda D Sharples, MRC Biostatistics Unit, Robinson Way, Cambridge, UK Nicholas W Morrell, University of Cambridge School of Clinical Medicine, Addenbrooke's Hospital, Cambridge, UK

This study had no financial support from external sources.

Competing interests: JS has received travel grants from Actelion Pharmaceuticals, Encysive, GlaxoSmithKline Beecham and Pfizer Ltd. RH has received travel grants, consulting fees and speaker's fees from Actelion Pharmaceuticals. KS has received travel grants from Actelion Pharmaceuticals. NM has received speaker's fees from Actelion Pharmaceuticals and received consulting fees from Novartis. JP-Z has received consulting fees from Actelion Pharmaceuticals, Pfizer Ltd and Encysive and research support from Actelion Pharmaceuticals and Pfizer Ltd. RM, LS, MT, DJ and RT have no relevant financial disclosures.

\section{REFERENCES}

1 Hoeper MM, Mayer E, Simonneau G, et al. Chronic thromboembolic pulmonary hypertension. Circulation 2006;113:2011-20.

2 Riedel M, Stanek V, Widimsky J, et al. Longterm follow-up of patients with pulmonary thromboembolism. Late prognosis and evolution of hemodynamic and respiratory data. Chest 1982;81:151-8.

3 Pengo V, Lensing AW, Prins $M H$, et al. Incidence of chronic thromboembolic pulmonary hypertension after pulmonary embolism. N Engl J Med 2004;350:2257-64

4 Jamieson SW, Kapelanski DP, Sakakibara N, et al. Pulmonary endarterectomy: experience and lessons learned in 1,500 cases. Ann Thorac Surg 2003;76:1457-4

5 Williamson TL, Kim NH, Rubin U. Chronic thromboembolic pulmonary hypertension. Prog Cardiovasc Dis 2002;45:203-12

6 Thistlethwaite PA, Mo M, Madani MM, et al. Operative classification of thromboembolic disease determines outcome after pulmonary endarterectomy. J Thorac Cardiovasc Surg 2002; 124:1203-11.

7 Simonneau G, Galie N, Rubin U, et al. Clinical classification of pulmonary hypertension. J Am Coll Cardiol 2004;43(12 Suppl S):5-12S.

8 Machado RD, Pauciulo MW, Thomson JR, et al. BMPR2 haploinsufficiency as the inherited molecular mechanism for primary pulmonary hypertension. Am J Hum Genet 2001;68:92-102.

9 Thomson JR, Machado RD, Pauciulo MW, et al. Sporadic primary pulmonary hypertension is associated with germline mutations of the gene encoding BMPR-II, a receptor member of the TGF-beta family. J Med Genet 2000;37:741-5.

10 Newman JH, Wheeler L, Lane KB, et al. Mutation in the gene for bone morphogenetic protein receptor II as a cause of primary pulmonary hypertension in a large kindred. N Engl J Med 2001;345:319-24.

11 Caslin AW, Heath D, Madden B, et al. The histopathology of 36 cases of plexogenic pulmonary arteriopathy. Histopathology 1990;16:9-19.

12 Moser KM, Fedullo PF, Finkbeiner WE, et al. Do patients with primary pulmonary hypertension develop extensive central thrombi? Circulation 1995;91:741-5.

13 Moser KM, Bloor CM. Pulmonary vascular lesions occurring in patients with chronic major vessel thromboembolic pulmonary hypertension. Chest 1993;103:685-92.

14 Egermayer P, Peacock AJ. Is pulmonary embolism a common cause of chronic pulmonary hypertension? Limitations of the embolic hypothesis. Eur Respir $J$ 2000;15:440-8.

15 Peacock AJ. Pulmonary hypertension after splenectomy: a consequence of loss of the splenic filter or is there something more? Thorax 2005;60:983-4.

16 Lewczuk J, Piszko P, Jagas J, et al. Prognostic factors in medically treated patients with chronic pulmonary embolism. Chest $2001 ; 119: 818-23$.

17 Coulden R. State-of-the-art imaging techniques in chronic thromboembolic pulmonary hypertension. Proc Am Thorac Soc 2006;3:577-83.

18 Remy-Jardin M, Remy J, Wattinne L, et al. Central pulmonary thromboembolism: diagnosis with spiral volumetric $\mathrm{CT}$ with the single-breath-hold techniquecomparison with pulmonary angiography. Radiology 1992;185:381-7.

19 Bergin CJ, Rios G, King MA, et al. Accuracy of high-resolution CT in identifying chronic pulmonary thromboembolic disease. AJR Am J Roentgenol 1996;166:1371-7.

20 Bonderman D, Jakowitsch J, Adlbrecht C, et al. Medical conditions increasing the risk of chronic thromboembolic pulmonary hypertension. Thromb Haemost 2005;93:512-6.

21 Fuster V, Steele PM, Edwards WD, et al. Primary pulmonary hypertension: natural history and the importance of thrombosis. Circulation 1984;70:580-7.

22 Jais $\mathbf{X}$, loos V, Jardim C, et al. Splenectomy and chronic thromboembolic pulmonary hypertension. Thorax 2005;60:1031-4.

23 Fedullo PF, Rubin $\sqcup$, Kerr KM, et al. The natural history of acute and chronic thromboembolic disease: the search for the missing link. Eur Respir J 2000;15:435-7

24 Du L, Sullivan CC, Chu D, et al. Signaling molecules in nonfamilial pulmonary hypertension. N Engl J Med 2003;348:500-9. 
25 Humbert M, Deng Z, Simonneau G, et al. BMPR2 germline mutations in pulmonary hypertension associated with fenfluramine derivatives. Eur Respir J 2002;20:518-23.

26 Roberts KE, McElroy JJ, Wong WP, et al. BMPR2 mutations in pulmonary arterial hypertension with congenital heart disease. Eur Respir J 2004;24:371-4.

27 Runo JR, Vnencak-Jones CL, Prince M, et al. Pulmonary veno-occlusive disease caused by an inherited mutation in bone morphogenetic protein receptor II. Am J Respir Crit Care Med 2003;167:889-94

28 Grant BJ, Lieber BB. Clinical significance of pulmonary arterial input impedance. Eur Respir J 1996:9:2196-9.

29 Burke CM, Glanville AR, Morris AJ, et al. Pulmonary function in advanced pulmonary hypertension. Thorax 1987;42:131-5.

30 Morris TA, Auger WR, Ysrael MZ, et al. Parenchymal scarring is associated with restrictive spirometric defects in patients with chronic thromboembolic pulmonary hypertension. Chest 1996;110:399-403.

31 Rich S, Dantzker DR, Ayres SM, et al. Primary pulmonary hypertension. A national prospective study. Ann Intern Med 1987;107:216-23.

32 Steenhuis LH, Groen HJ, Koeter GH, et al. Diffusion capacity and haemodynamics in primary and chronic thromboembolic pulmonary hypertension. Eur Respir J 2000;16:276-81.
33 Bonderman D, Nowotny R, Skoro-Sajer N, et al. Bosentan therapy for inoperable chronic thromboembolic pulmonary hypertension. Chest 2005;128:2599-603.

34 Ghofrani HA, Schermuly RT, Rose F, et al. Sildenafil for long-term treatment of nonoperable chronic thromboembolic pulmonary hypertension. Am J Respir Crit Care Med 2003; 167:1139-41.

35 Ghofrani HA, Wiedemann R, Rose F, et al. Combination therapy with oral sildenafil and inhaled iloprost for severe pulmonary hypertension. Ann Intern Med 2002; 136:515-22.

36 Hoeper MM, Kramm T, Wilkens H, et al. Bosentan therapy for inoperable chronic thromboembolic pulmonary hypertension. Chest 2005;128:2363-7.

37 Hughes R, George P, Parameshwar J, et al. Bosentan in inoperable chronic thromboembolic pulmonary hypertension (letter). Thorax 2005;60:707.

38 Hughes RJ, Jais X, Bonderman D, et al. The efficacy of bosentan in inoperable chronic thromboembolic pulmonary hypertension: a 1-year follow-up study. Eur Respir J 2006;28: 138-43.

39 D'Alonzo. Survival in patients with primary pulmonary hypertension: results from a national prospective registry. Ann Intern Med 1991;115:343-9.

40 Hodgkins D, Sharples LC, Fowler SJ, et al. Survival in pulmonary hypertension the Papworth experience. Amercian Thoracic Society Conference 2005:A802.

\section{PULMONARY PUZZLE}

\section{Prolonged fever, dyspnoea and diffuse pleural thickening in a 20-year-old man}

\section{Clinical presentation}

A 20-year-old man was referred for intermittent high fever, night sweat and loss of body weight for 6 months associated with progressive exertional dyspnoea which developed 4 weeks before admission. The patient was unresponsive to antibiotics and 14 day pre-emptive antituberculous chemotherapy with rifampicin, isoniazid, ethambutol and pyrazinamide. His temperature was $39.8^{\circ} \mathrm{C}$, heart rate 96 beats $/ \mathrm{min}$, blood pressure $110 / 70 \mathrm{~mm} \mathrm{Hg}$, breath rate $22 / \mathrm{min}$ with decreased breath sounds in the left upper thorax without cyanosis, clubbing and hepatosplenomegaly.

Laboratory examinations showed mild microcytic hypochromic anaemia, erythrocyte sedimentation rate $46 \mathrm{~mm} / \mathrm{h}$, severe restrictive airflow limitation with impairment of transfer factor. Blood biochemistry including angiotensin converting enzyme (ACE), electrolytes including calcium, immunological profiles including antinucleic antibody, extractable nuclear antigens, antineutrophil cytoplasmic antibody, and HIV antibody, tuberculin skin test, Ziehl-Neelson stain, repeated blood culture, abdomen ultrasonography and fibrobronchoscopy were unremarkable.

Plain chest radiography showed bilateral pleural thickening without parenchymal abnormality. A high resolution CT
(HRCT) scan of the chest displayed thickening of the mediastinal, lateral and interlobar pleura (some $>1 \mathrm{~cm}$ in thickness), multilobar subpleural pulmonary micronodules and thickening of bronchovascular bundles without intrathoracic adenopathy (fig 1A, B).

\section{Question}

What is the likely diagnosis and how should it be confirmed? See page 637

This case was submitted by:

C Cai

Department of Pulmonology, General Hospital of Guangzhou Military Command, Guangzhou, China D R Li, Q S Zeng, S Q Zhong, N S Zhong Guangzhou Institute of Respiratory Disease, First Affiliated Hospital of Guangzhou Medical College, Guangzhou, China

Correspondence to: Dr Chuang Cai, Department of Pulmonology, General Hospital of Guangzhou Military Command, No 111 Liuhua Rd, Guangzhou, China 510010; skinblack1966@ yahoo.com.cn

doi: $10.1136 /$ thx.2007.081232
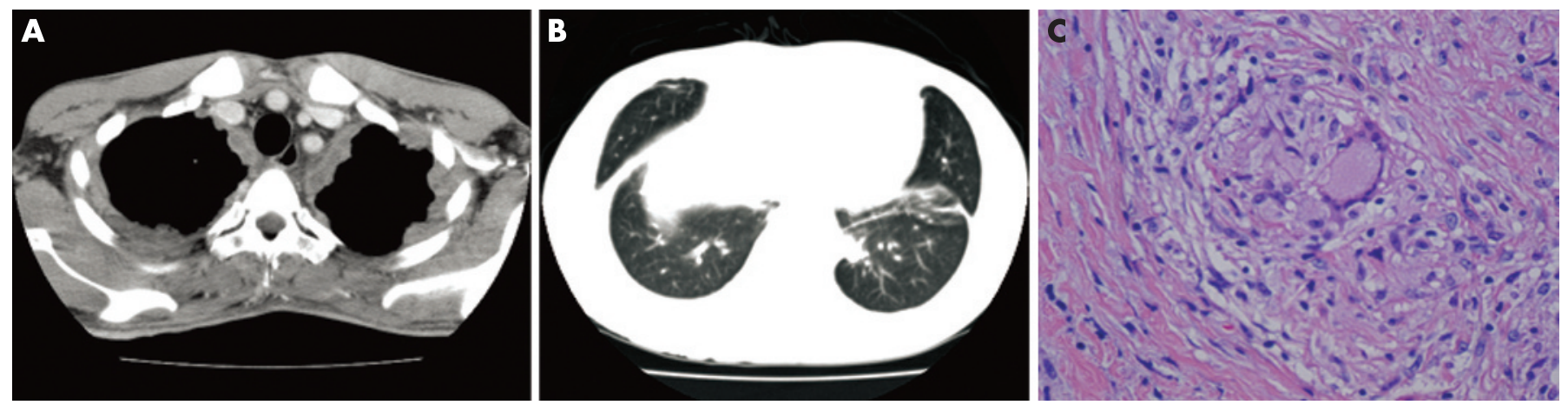

Figure 1 (A) High resolution CT (HRCT) scan showing diffuse thickening of lateral and mediastinal pleura with pleural nodularity, some $>1 \mathrm{~cm}$ in thickness. (B) HRCT scan showing diffuse subpleural micronodules, thickening of bronchovascular bundles and thickening of interlobar septa. (C) Histological examination showing non-caseating epithelioid granuloma with focal central necrosis in biopsied pleura (H\&E; $\times 400)$. 